\title{
Segment phasing experiments on the High Order Test bench
}

\author{
E. Aller-Carpentier ${ }^{1, a}$, M. Kasper ${ }^{1}$, and P. Martinez ${ }^{1}$ \\ ESO, Karl-Schwarzschild-Strasse 2, 85748 Garching bei Muenchen, Germany.
}

\begin{abstract}
The segmented primary mirror of the E-ELT imposes particular requirements on an Extreme Adaptive Optics (XAO) system. At present, there are already several AO systems working on segmented telescopes but the achieved performances are too low to draw conclusions for XAO systems aiming at some $90 \%$ Strehl ratio in the NIR. On other hand, several analytical studies and simulations were done, but laboratory studies are required to confirm the corrections expected. The goal of the present study is to determina the capability of XAO systems to deal with segmentation piston errors. In particular, the effects on the AO performance and the ability of the AO system to correct the segmentation piston errors were studied. The experiments were carried out on the High Order Test Bench at ESO (Munich) using a Shack-Hartmann wave front sensor and under most realistic conditions with phase screens simulating atmospheric turbulence and segmentation piston errors. Segment geometry was chosen such that about 6 actuators of the XAO DM cover one segment representing the design of EPICS at the EELT.
\end{abstract}

\section{Introduction}

Extreme Adaptive Optics (XAO) at the E-ELT requires dealing with a primary segmented mirror. The wave front control of the ELT includes three main units: adaptive optics, active optics and phasing camera. Each wave front control is affected to some extend by the total wavefront, but, on the other hand the different control units can "help" each other. The wavefront sensor of the AO unit will see the telescope aberrations and segments misalignments; hence the AO deformable mirror will try to compensate for them.

The goal of the present study is to determina the capability of the adaptive optics to deal with and correct for the segmentation piston errors. At present, there are several segmented telescope working with $\mathrm{AO}$ systems but the experience from them could not be use for extrapolation to a XAO system. Several analytical studies and simulations were done [2]. From these studies, the ability of the AO system to correct for the segmentation errors strictly depends on the design of the DM and the wave front sensor. The density of the actuators, the coupling factor and the geometry of actuators distribution have directly impact on the performance.

The ELT primary mirror will be composed by around 1000 hexagonal segments of $1.5 \mathrm{~m}$ with a gap of $10 \mathrm{~mm}$. 5-7 actuators per segment are expected considering a deformable mirror of 200x200 actuators. The experiments were carried out on the HOT Bench using a Shack-Hartman WFS and taking into account the ELT geometry and the requirements for a XAO instrument. They will also be run under realistic atmospheric conditions.

\section{HOT: The High Order Test Bench}

"The High Order Test bench" [1], [3] is an experimental bench for extreme adaptive optics (XaO) systems optimization. Realistic conditions are achieved on the bench simulating the VLT pupil (8m) with a F/48 beam and applying different pupil masks. The HOT bench incorporates a turbulence generator with phase screens to simulate real seeing conditions.

Two deformable mirrors for wavefront correction are integrated in the system. A first deformable mirror (60 bimorph elements) installed on a Tip Tilt Mount is used to correct for static aberrations.

a e-mail: eallerca@eso.org

This is an Open Access article distributed under the terms of the Creative Commons Attribution-Noncommercial License, which permits unrestricted use, distribution, and reproduction in any noncommercial medium, provided the original work is properly cited. 

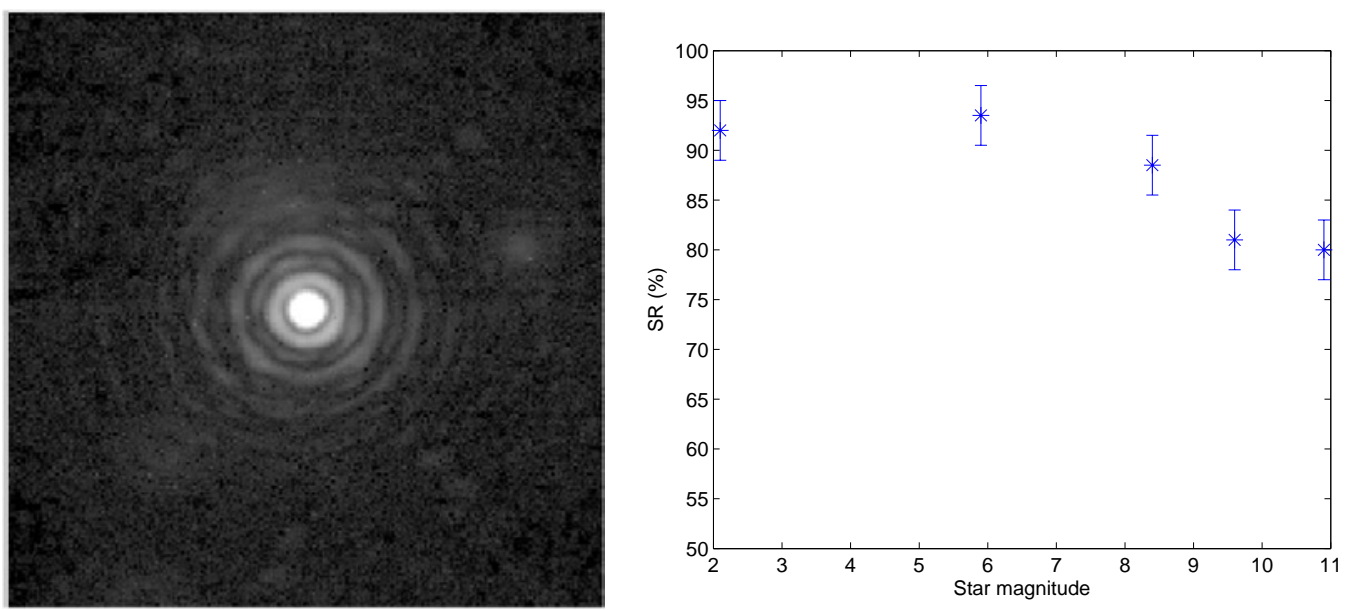

Fig. 1. Left: PSF image obtained for high flux condition (mag 6), SR $>90 \%$, using a 600 modes reconstructor. Right: SR performance as a function of the star magnitude (a 3\% of error was assumed for the SR estimation)

The second one is a $32 \times 32$ micro deformable mirror (electrostatic MEMS device from Boston micromachines, $340 \mu \mathrm{m}$ pitch - $1.53 \mu \mathrm{m}$ mechanical stroke) to correct for the high order modes of the generated turbulence.

A cube beamsplitter splits the optical beam in two channels. A Shack-Hartmann or a pyramid [6] working on the visible can be chosen on the first channel for wave front sensing. The SHS works with an $\mathrm{F} / 48$ input beam and provides a plate scale of $0.5 \mathrm{arcsec} /$ pixel, with $31 \times 31$ subapertures, each detected on 4 x 4 pixels. Both sensors use a sub-electron readout noise Andor iXON L3 CCD of 24 $\mu \mathrm{m}$ pixel.

In the IR channel the scientific image is studied using the ESO IR Test Camera (1 kx $1 \mathrm{k}$ HAWAII detector - 5.3 mas per pixel - , cooled at $106 \mathrm{~K}$ with a vacuum of $10^{-5}$ mbar). Different types of coronagraph (APLC, Lyot, FQPM, Band limited) are available for high contrast imaging.

\subsection{Closed loop SHS performance}

A simple antiwind-up integrator algorithm is implemented for the closed loop. The BMM and the TTM are controlled using a modal reconstructor ( 600 modes) obtained as a combination of a zonal interaction matrix and a modal base. A Hadamard matrix is used to maximize the $\mathrm{S} / \mathrm{N}$ on the zonal calibration. The modal base is constructed as a Karhunen-Loève base where the modes are orthogonal and uncorrelated taking in account the atmospheric profile.

A quadratic control law is implemented to control the BMM since the electrostatic actuators behavior are not linear. 800 active actuators are controlled plus an external ring of slave actuators used to increase the stroke of the edge pupil actuators.

The SR achieved in $\mathrm{H}$ band is $>90 \%$ at high flux (mag 5) and $80 \%$ at lower flux (mag 10-11) in 0.5 " seeing with low order aberrations reduced to avoid DM saturations (figure 1b). The corrected PSF is shown on figure 1a).

\subsection{XAO with APLC coronagraph}

An APLC coronagraph specifically optimized for HOT characteristics $(2.3 \lambda / D$ inner working angle, i.e. 90 mas) is integrated in the scientific path. This coronagraph combines a pupil apodization with an hard-edged focal plane occultor (Lyot mask, $4.5 \lambda / D$ ) and an undersized pupil-plane stop (90\%). 

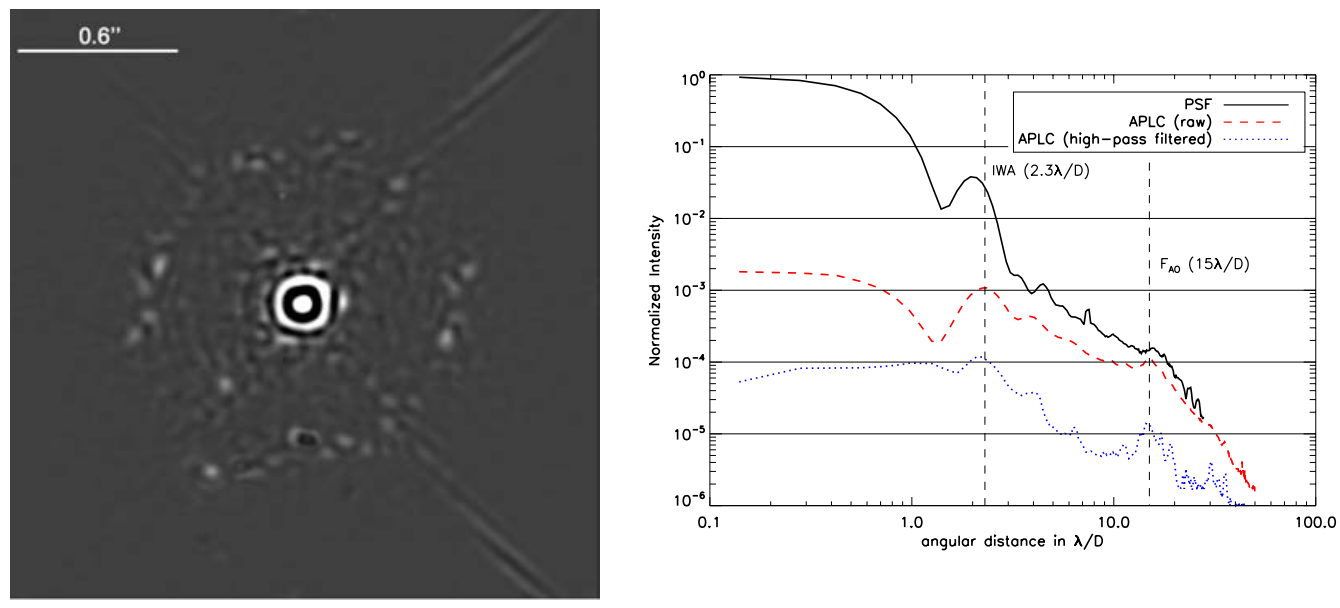

Fig. 2. Left: High-pass filtered APLC image in H-band. Right: Azimuthally averaged contrast profiles: PSF (black), APLC raw image (red) and high-pass filtered APLC image (blue, $1 \sigma$ contrast).

Figure 2 shows the high-pass filtered coronagraphic image at $1.6 \mu \mathrm{m}$ (bandwith of $64 \mathrm{~nm}$, i.e. $\Delta \lambda / \lambda=4 \%$ ). The image shows the cut-off frequency of the AO-correction domain at $15 \lambda / D$ i.e. 0.6". The contrasts obtained are $(1 \sigma): 1.1 \times 10^{-4}$ at IWA (90 mas), $5 \times 10^{-5}$ at 0.1 ", $1.4 \times 10^{-5}$ at 0.2 " $(5 \lambda / D)$, and $4.5 \times 10^{-6}$ at $0.5 "(12 \lambda / D)$. These level of contrast are in good agreement with SPHERE near-IR instrument simulation predictions [7]. Further details of the APLC design and performance could be found in [4], [5].

\section{The cophasing experiment}

The residual piston phase error on the EELT after segment cophasing correction is simulated on the HOT bench using a Piston Phase Plate (PPP). Thus, the impact on the AO performance and the residual error on the PSF could be studied. Since the segmentation should be studied in the pupil plane, the PPP is installed on the turbulence generator replacing one of the turbulence phase screen.
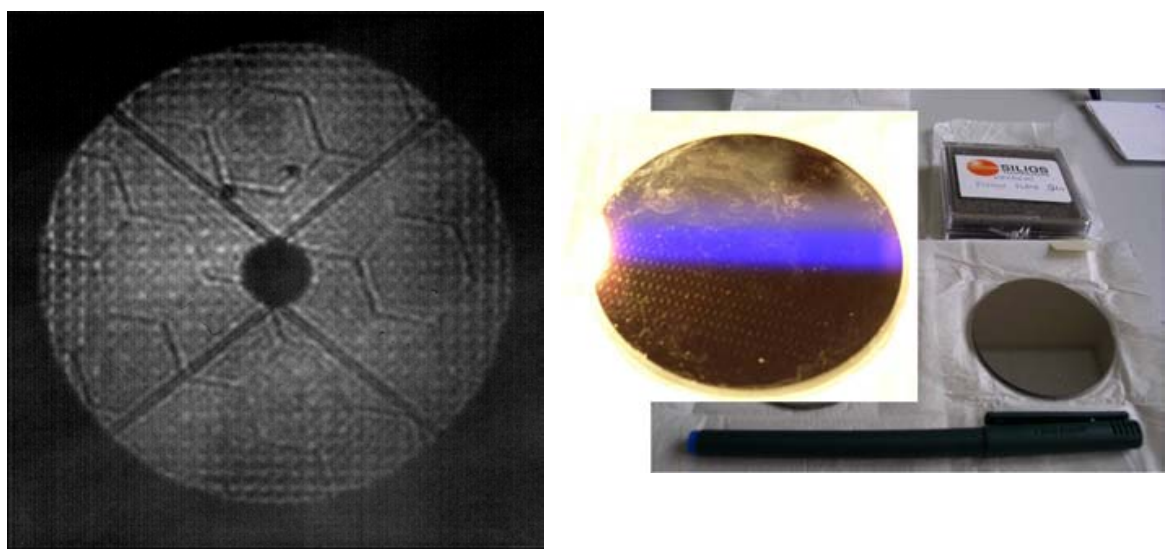

Fig. 3. Left:Pupil plane image showing the segmentation Right:Piston phase plate (PPP) 
19 hexagonal segments cover the equivalent pupil on the PPP $(6.7 \mathrm{~mm})$, providing an actuator/segment rate of 6-7 actuators as expected for the XAO instrument at the E-ELT. Segments are distributed at 4 piston levels $(0,15,35,75 \mathrm{~nm})$ providing an rms wave-front error of $52 \mathrm{~nm}$ (EPICS specification on EELT is $36 \mathrm{~nm} \mathrm{rms}$ ). The complete PPP disk of $50 \mathrm{~mm}$ diameter is covered by segments and can rotate on its mount, offering the possibility of studying different alignments between segments and actuators.

Now the strength of the turbulence will decreased since only one of the two phase screens is used. An effective seeing of 0.4 " will be used on the experiments with turbulence.

\subsection{Segmentation effects}

The impact of the PPP on the AO performance was studied on closed loop (with turbulence) analyzing the PSF and SHS slopes rms. The performance did not differ from the results obtained on section 2 achieving a SR of $90 \%$ (using the same AO parameters). To see the effect of the segmentation it was necessary to increase the contrast. Hence, coronagraphic images were acquired with and without turbulence and with and without segmentation for comparison purpose (figure 4)).
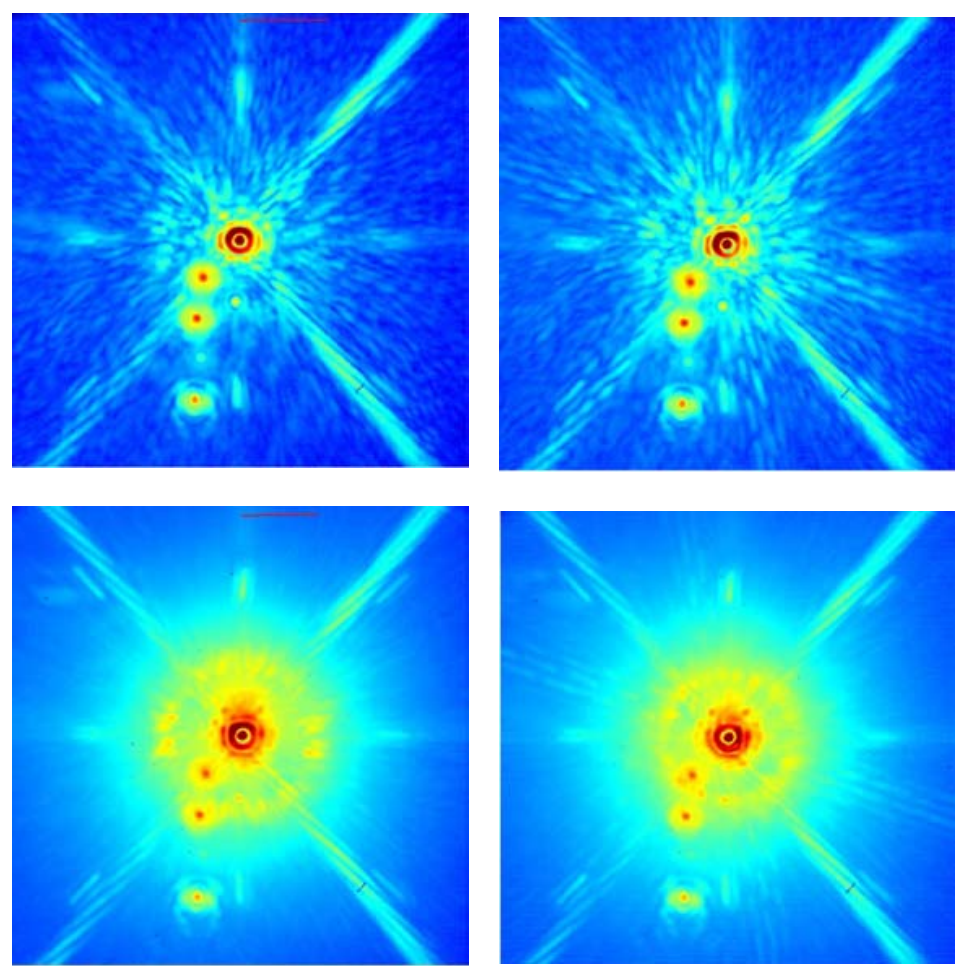

Fig. 4. Up: Coronagraphic images without (right) and with (left) Piston Phase Plate in absence of turbulence. Down: Same cases but in presence of turbulence. In the PPP cases an addtional diffraction pattern with three axes at 60 degrees is observed.

The effects of the segmentation can be seen only outside the AO control frequency region (> $15 \lambda / D$ ) on the coronagraphic images. The resulted images show the diffraction pattern with three axes at 60 degrees observed at a contrast level between $3.5 \times 10^{-5}$ and $8 \times 10^{-6}$. Azymuthally averaged contrast profiles for the studied cases are presented in figure figure 5. The profiles show that the contrast reached is not affected by the PPP. In both cases, three images were recorded with different segments 
E. Aller-Carpentier et al.: Segment phasing experiments on the High Order Test bench

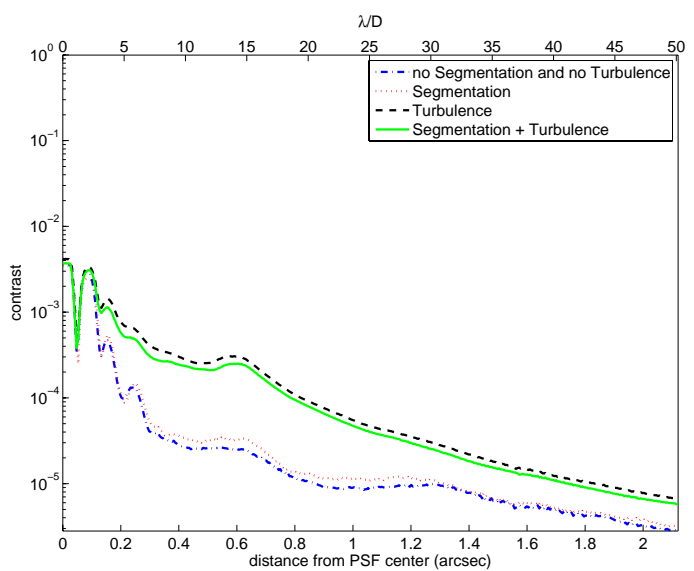

Fig. 5. Contrast curves for the 4 studied cases (PPP and no PPP, with and whithout turbulence). The contrast reached is not affected by the PPP.

orientations (figure 6). The original image without segmentation has then been subtracted to identify the segmentation effect. As expected, a rotation on the diffraction pattern produced by the segments can be seen (all images were acquired on closed loop to avoid internal turbulence).
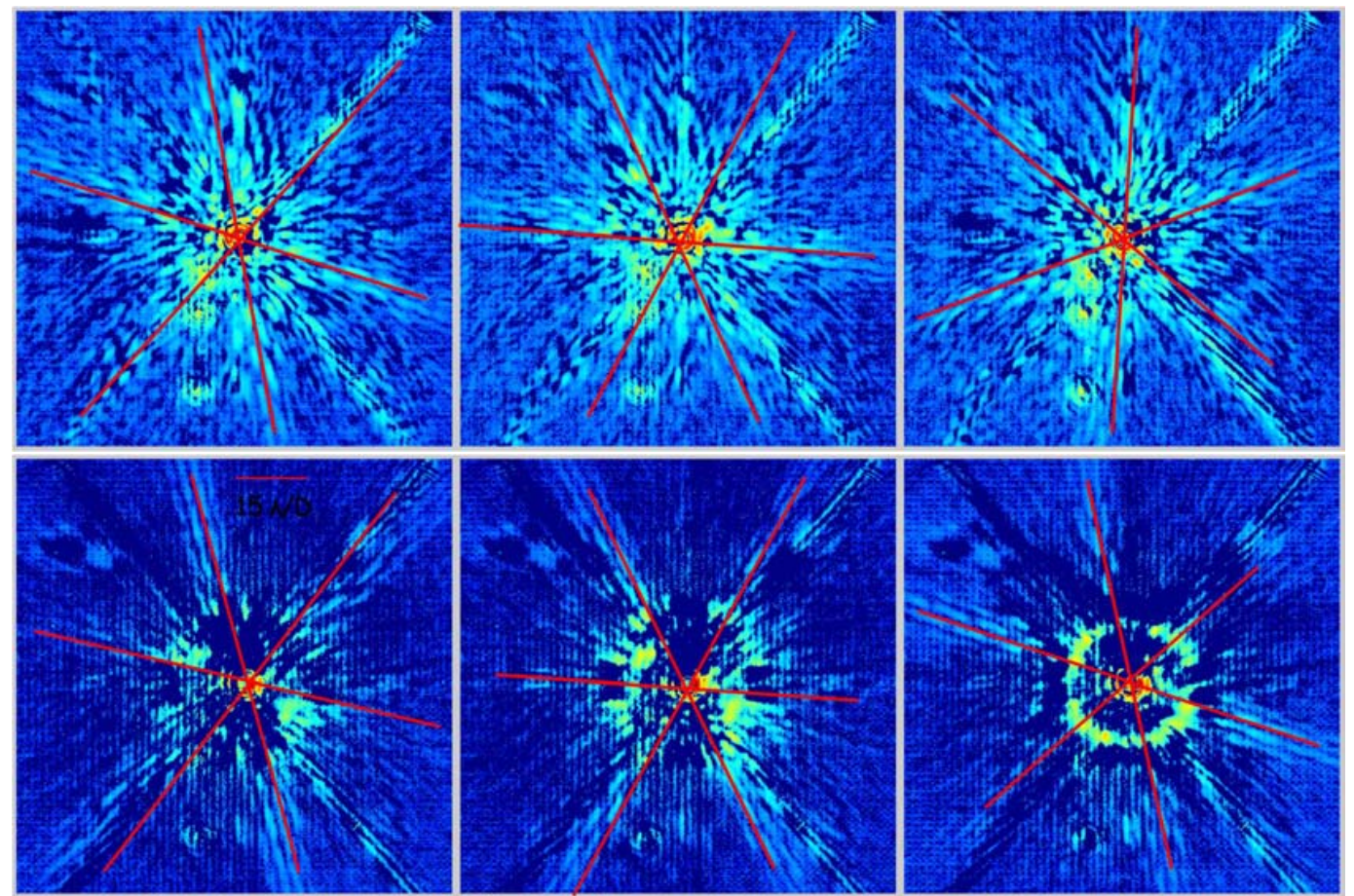

Fig. 6. Up: Subtracted coronagraphic PPP images from the non segmented case in absence of turbulence. The three images corresponds to different segments orientations. Down: Same cases but in presence of turbulence. As expected, a rotation on the diffraction pattern produced by the segments can be observed. 
Coronagraphic images were simulated taking into account the effect of the segmentation following the PPP geometry (19 segments - $52 \mathrm{~nm} \mathrm{rms).} \mathrm{The} \mathrm{segmentation} \mathrm{effect} \mathrm{is} \mathrm{observed} \mathrm{at} \mathrm{contrast} \mathrm{level}$ around $5 \times 10^{-6}-5 \times 10^{-7}$, being in agreement with the experimental data (assuming no other error sources).

\section{Conclusion}

The residual piston phase error after EELT segment cophasing correction is simulated on real AO conditions using a piston phase plate and assuming $52 \mathrm{~nm} \mathrm{rms}$ of piston error. The preliminary results demonstrate that the $\mathrm{AO}$ system is not disturbed and the performance achieved is similar to that without PPP (> 90\% SR). The effect of the segmentation is only observed at contrast levels of $10^{-5}-10^{-6}$ outside the AO control area (i.e., not corrected by the AO system). This results are on good agreement with the simulations.

To know whether the AO system is able to correct the segmentation error it is necessary to look inside the AO control area. The contrast level in this area is limited by speckle, being approximatelly one order of magnitude higher than the segmentation effect. Speckle nulling techniques are foreseen in order to achieve the contrast level required.

\section{Acknowledgement}

The activity outlined in this paper was supported by the European Commission under the FP6-JRA1OPTICON contract number RII3-CT-2004-001566 and Seventh Framework Programme (FP7 - WP 9000), Capacities Specific Programme, Research Infrastructures; specifically the FP7, Preparing for the construction of the European Extremely Large Telescope Grant Agreement, Contract number INFRA-2007-2.2.1.28.

\section{References}

1. Aller-Carpentier, E. and Kasper, M. and Martinez, P. and Vernet, E. and Fedrigo, E. and Soenke, C. and Tordo, S. and Hubin, N. and Verinaud, C. and Esposito, S. and Pinna, E. and Puglisi, A. and Tozzi, A. and Quiros, F. and Basden, A. G. and Goodsell, S. J. and Love, G. D. and Myers, R. M., Society of Photo-Optical Instrumentation Engineers (SPIE) Conference Series 7015, (2008) $70153 Z$

2. Yaitskova, N. and Verinaud, C., Society of Photo-Optical Instrumentation Engineers (SPIE) Conference Series 6267, (2006) 62672N

3. Vernet, E. and Kasper, M. and Vérinaud, C. and Fedrigo, E. and Tordo, S. and Hubin, N. and Esposito, S. and Pinna, E. and Puglisi, A. and Tozzi, A. and Basden, A. G. and Goodsell, S. J. and Love, G. D. and Myers, R. M., Society of Photo-Optical Instrumentation Engineers (SPIE) Conference Series 6272, (2006) 62722K

4. Martinez, P. and Vernet, E. and Dorrer, C. and Aller Carpentier, E. and Boccaletti, A. and Kasper, M. and Baudrand, J. and Chaumont, C., Society of Photo-Optical Instrumentation Engineers (SPIE) Conference Series 7015, (2008) 70156B

5. Martinez, P. and Dorrer, C. and Aller Carpentier, E. and Kasper, M. and Boccaletti, A. and Dohlen, K. and Yaitskova, N., A\&A 495, (2009) 363-370

6. Pinna, E. and Puglisi, A. T. and Quiros-Pacheco, F. and Busoni, L. and Tozzi, A. and Esposito, S. and Aller-Carpentier, E. and Kasper, M., Society of Photo-Optical Instrumentation Engineers (SPIE) Conference Series 7015, (2008) 701559

7. Boccaletti, A. and Carbillet, M. and Fusco, T. and Mouillet, D. and Langlois, M. and Moutou, C. and Dohlen, K., Society of Photo-Optical Instrumentation Engineers (SPIE) Conference Series 7015, (2008) 70156E 